VoL. $62(2000) \quad[1-19]$

\title{
COMPOSITION OPERATORS ON SOME MÖBIUS INVARIANT BANACH SPACES
}

\author{
Shamil Makhmutov and Maria TJani
}

\begin{abstract}
We characterise the compact composition operators from any Mobius invariant Banach space to VMOA, the space of holomorphic functions on the unit disk $U$ that have vanishing mean oscillation. We use this to obtain a characterisation of the compact composition operators from the Bloch space to VMOA. Finally, we study some properties of hyperbolic VMOA functions. We show that a function is hyperbolic VMOA if and only if it is the symbol of a compact composition operator from the Bloch space to VMOA.
\end{abstract}

\section{INTRODUCTION}

Let $\phi$ be a holomorphic self-map of the open unit disc $U$, and $H^{2}$ the Hilbert space of functions holomorphic on $U$ with square summable power series coefficients. Associate with $\phi$ the composition operator $C_{\phi}$ defined by

$$
C_{\phi} f=f \circ \phi
$$

for $f$ holomorphic on $U$.

This is the first setting in which composition operators were studied. By Littlewood's Subordination Principle every composition operator takes $H^{2}$ into itself. Shapiro in [16], using the Nevanlinna counting function, characterised the compact composition operators on the Hardy space $H^{2}$. A natural follow up question is about the boundedness and compactness of composition operators on other function spaces. We know the answer to this question in a variety of spaces.

Madigan and Matheson show in [10] that $C_{\phi}$ is compact on the Bloch space $\mathcal{B}$ if and only if

$$
\lim _{|\phi(z)| \rightarrow 1}\left(1-|z|^{2}\right) \frac{\left|\phi^{\prime}(z)\right|}{1-|\phi(z)|^{2}}=0 .
$$

In this paper we study operators which map certain subspaces of the Bloch space into BMOA and into VMOA. Some other approaches to this problem are studied in $[5,18,19]$.

Received 6th July, 1999

Copyright Clearance Centre, Inc. Serial-fee code: 0004-9727/00 \$A2.00+0.00. 
In Section 2 we give some preliminaries. In Section 3 we give a sufficient condition for the compactness of $C_{\phi}: \mathcal{B} \rightarrow \mathrm{BMOA}$. In Section 4 we give a characterisation of compact operators $C_{\phi}: X \rightarrow \mathrm{VMOA}$, where $X$ is a Möbius invariant subspace of the Bloch space. We use this characterisation to show that the condition

$$
\lim _{|q| \rightarrow 1} \int_{U} \frac{\left|\phi^{\prime}(z)\right|^{2}\left(1-\left|\alpha_{q}(z)\right|^{2}\right)}{\left(1-|\phi(z)|^{2}\right)^{2}} d A(z)=0
$$

is necessary and sufficient for the compactness of $C_{\phi}: \mathcal{B} \rightarrow$ VMOA.

In Section 5 we consider the case when $\phi$ is a boundedly valent holomorphic selfmap of $U$ such that $\phi(U)$ lies inside a polygon inscribed in the unit circle and observe a similarity of compactness conditions for the Bloch space, BMOA and VMOA (see Theorem 5.3). It is a corollary of this theorem (see corollary 5.4) that if $C_{\phi}$ is a weakly compact operator on VMOA then it is a compact operator, if $\phi(U)$ lies inside a polygon inscribed in the unit circle.

Let $\sigma(a, b)$ be the hyperbolic metric on $U$

$$
\begin{gathered}
\sigma(a, b)=\frac{1}{2} \log \frac{1+\rho(a, b)}{1-\rho(a, b)} \\
\rho(a, b)=\left|\frac{a-b}{1-\bar{a} b}\right|
\end{gathered}
$$

and $D(a, r)=\{z \in U: \rho(a, z) \leqslant r\}$ be a pseudohyperbolic disk with a centre $a \in U$ and a radius $r, 0<r<1$. In the last section we study the hyperbolic VMOA functions $\left(\mathrm{VMOA}_{h}\right)$, that is, holomorphic self-maps $\phi$ of the unit disk $U$ such that

$$
\lim _{|q| \rightarrow 1} \int_{\partial U} \sigma\left(\phi \circ \alpha_{q}(z), \phi(q)\right)|d z|=0
$$

It is proved (Theorem 6.1) that $\phi \in \mathrm{VMOA}_{h}$ if and only if $\phi$ satisfies condition (1). Some properties of hyperbolic VMOA functions are studied.

\section{Preliminaries}

Let $U$ be the open unit disc in the complex plane $\mathbf{C}$ and $\partial U$ be the unit circle. The one-to-one holomorphic functions that map $U$ onto itself, called the Möbius transformations, and denoted by $G$, have the form $\lambda \alpha_{p}$ where $\lambda \in \partial U$ and $\alpha_{p}$ is the basic conformal automorphism defined by $\alpha_{p}(z)=(p-z) /(1-\bar{p} z)$ for $p \in U$. It is easy to check that the inverse of $\alpha_{p}$ under composition is $\alpha_{p}$

$$
\alpha_{p} \circ \alpha_{p}(z)=z
$$

for $z \in U$. Also,

$$
\left|\alpha_{p}^{\prime}(z)\right|=\frac{1-|p|^{2}}{|1-\bar{p} z|^{2}}
$$


and

$$
1-\left|\alpha_{p}(z)\right|^{2}=\frac{\left(1-|p|^{2}\right)\left(1-|z|^{2}\right)}{|1-\bar{p} z|^{2}}=\left(1-|z|^{2}\right)\left|\alpha_{p}^{\prime}(z)\right|
$$

for $p, z \in U$.

The Bloch space $\mathcal{B}$ on $U$ is the space of holomorphic functions $f$ on $U$ such that

$$
\|f\|_{\mathcal{B}}=\sup _{z \in U}\left(1-|z|^{2}\right)\left|f^{\prime}(z)\right|<\infty
$$

The norm $\|f\|=|f(0)|+\|f\|_{B}$ makes the Bloch space a Banach space. Using (2) one can see that $\mathcal{B}$ is invariant under Möbius transformations, that is, if $f \in \mathcal{B}$ then $f \circ \phi \in \mathcal{B}$, for all $\phi \in G$. In fact,

$$
\|f \circ \phi\|_{B}=\|f\|_{B} .
$$

We note that the polynomials are not dense in the Bloch space and the closure of the polynomials in the Bloch norm is the little Bloch space, denoted by $\mathcal{B}_{0}$. In [22, p.84] it is shown that

$$
f \in \mathcal{B}_{0} \text { if and only if } \lim _{|z| \rightarrow 1}\left(1-|z|^{2}\right)\left|f^{\prime}(z)\right|=0
$$

A linear space $X$ of holomorphic functions on $U$ with a seminorm $\|.\|_{X}$ is Möbius invariant if

1. $X \subseteq \mathcal{B}$ and there exists a positive constant $c$ such that for all $f \in X$,

$$
\|f\|_{B} \leqslant c\|f\|_{X}
$$

2. For all $\phi \in G$ and all $f \in X, f \circ \phi \in X$ and

$$
\|f \circ \phi\|_{x}=\|f\|_{x}
$$

A Möbius invariant Banach space $X$ is a Möbius invariant linear space of holomorphic functions on $U$ with a seminorm $\|\cdot\|_{X}$, whose norm is $\|f\|_{X}$ or $|f(0)|+\|f\|_{X}$. Some examples of Möbius invariant Banach spaces include the Besov spaces, VMOA, BMOA and the Bloch space. In fact, the reason for insisting that a Möbius invariant Banach space be a subspace of the Bloch space is that Rubel and Timoney proved in [14] that if a linear space of analytic functions on $U$ with a seminorm $\|.\|_{X}$ is such that for all $f \in X, f \circ \phi \in X$ and $\|f \circ \phi\|_{X}=\|f\|_{X}$, and it has a non-zero linear functional $L$ that is decent (that is $L$ extends to a continuous linear functional on the space of holomorphic functions on $U$ ) then, $X$ has to be a subspace of the Bloch space and the inclusion map is continuous.

A holomorphic function $f$ on $U$ belongs to BMOA, the holomorphic members of $\mathrm{BMO}$, if

$$
\|f\|_{\mathrm{BMOA}}=\sup _{q \in U}\left\|f \circ \alpha_{q}(z)-f(q)\right\|_{H^{2}}<\infty
$$


Under the norm $|f(0)|+\|f\|_{\mathrm{BMOA}}$ the space BMOA becomes a complete normed linear space. This is not the traditional definition of BMOA, it is actually a corollary of the John-Nirenberg theorem [4, p.15]. By the Littlewood-Paley identities (see [22, p.167]) and the fact that $\log 1 /|z| \sim 1-|z|^{2}$, for $z$ away from the origin we see that a seminorm equivalent to the one defined in (3) is:

$$
\begin{aligned}
\|f\|_{*}^{2} & =\sup _{q \in U} \int_{U}\left|\left(f \circ \alpha_{q}\right)^{\prime}(z)\right|^{2}\left(1-|z|^{2}\right) d A(z) \\
& =\sup _{q \in U} \int_{U}\left|f^{\prime}\left(\alpha_{q}(z)\right)\right|^{2}\left|\alpha_{q}^{\prime}(z)\right|^{2}\left(1-|z|^{2}\right) d A(z) .
\end{aligned}
$$

Thus after the change of variables $\alpha_{q}(z)=w$ we obtain

$$
\|f\|_{*}^{2}=\sup _{q \in U} \int_{U}\left|f^{\prime}(w)\right|^{2}\left(1-\left|\alpha_{q}(w)\right|^{2}\right) d A(w) .
$$

Let const. denote a positive constant which may change from one occurrence to the next but will not depend on the functions involved. Let $A$ and $B$ be two quantities that depend on a holomorphic function $f$ on $U$. We say that $A$ is equivalent to $B$, and we write $A \sim B$, if

$$
\text { const. } A \leqslant B \leqslant \text { const. } A \text {. }
$$

Let $S(h, \theta)=\left\{z \in U:\left|z-e^{i \theta}\right|<h\right\}$, where $\theta \in[0,2 \pi), h \in(0,1)$.

The notion of BMOA first arose in the context of mean oscillations of a function over cubes with edges parallel to the coordinate axes or equivalently over sets of the form $S(h, \theta)[15$, pp.36-39]. That is,

$$
\|f\|_{*}^{2} \sim \sup _{\substack{h \in(0,1) \\ \theta \in[0,2 \pi)}} \frac{1}{h} \int_{S(h, \theta)}\left|f^{\prime}(z)\right|^{2}\left(1-|z|^{2}\right) d A(z) .
$$

One of the many similarities between the Bloch space and BMOA is that polynomials are not dense in either space. The closure of polynomials in the BMOA norm forms VMOA, the space of holomorphic functions with vanishing mean oscillation. The space VMOA can be characterised as all those holomorphic functions $f$ on $U$ such that

$$
\lim _{|q| \rightarrow 1} \int_{V}\left|f^{\prime}(w)\right|^{2}\left(1-\left|\alpha_{q}(w)\right|^{2}\right) d A(w)=0
$$

(the "little-oh" version of (5)). Moreover the "little-oh" version of (6) is equivalent to (7) $[15$, pp.36-39, p.50].

Recall another characterisation of BMOA and VMOA functions involving Carleson measure. A positive measure $\mu$ on $U$ is a Carleson measure provided that

$$
\nu(\mu)=\sup _{h, \theta} \frac{\mu(S(\theta, h))}{h}<\infty .
$$


By Fefferman's theorem [9], a function $f \in$ BMOA if and only if

$$
d \mu_{f}(z)=\left|f^{\prime}(z)\right|^{2}\left(1-|z|^{2}\right) d x d y
$$

is a Carleson measure on $U$. Note that by (6)

$$
\|f\|_{*}^{2} \sim \nu\left(\mu_{f}\right)
$$

With regard to VMOA functions, we can say that a function $f \in \mathrm{BMOA}$ is in VMOA if and only if

$$
\lim _{h \rightarrow 0} \frac{\mu_{f}(S(\theta, h))}{h}=0
$$

(see [9]), that is, $\mu_{f}$ is a compact Carleson measure [3].

The counting function for BMOA is defined by

$$
N(w, q, \phi)=\sum_{\phi(z)=w}\left(1-\left|\alpha_{q}(z)\right|^{2}\right)
$$

for $w, q \in U$. Then

$$
\begin{aligned}
\left\|C_{\phi} f\right\|_{*}^{2} & =\sup _{q \in U} \int_{U}\left|(f \circ \phi)^{\prime}(z)\right|^{2}\left(1-\left|\alpha_{q}(z)\right|^{2}\right) d A(z) \\
& =\sup _{q \in U} \int_{U}\left|f^{\prime}(\phi(z))\right|^{2}\left|\phi^{\prime}(z)\right|^{2}\left(1-\left|\alpha_{q}(z)\right|^{2}\right) d A(z) .
\end{aligned}
$$

By making a non-univalent change of variables as is done in $[17, p .186]$ we see that

$$
\left\|C_{\phi} f\right\|_{*}^{2}=\sup _{q \in U} \int_{U}\left|f^{\prime}(w)\right|^{2} N(w, q, \phi) d A(w)
$$

Throughout this paper, $B$ will denote the set of holomorphic self-maps $\phi$ of the unit disk $U$. By the Schwarz-Pick lemma [9], for every $\phi \in B$

$$
\sup _{z \in U}\left(1-|z|^{2}\right) \frac{\left|\phi^{\prime}(z)\right|}{1-|\phi(z)|^{2}} \leqslant 1 .
$$

We say that $\phi \in B_{0}$ if

$$
\lim _{|z| \rightarrow 1}\left(1-|z|^{2}\right) \frac{\left|\phi^{\prime}(z)\right|}{1-|\phi(z)|^{2}}=0
$$

\section{BLOCH-TO-BMOA COMPOSITION OPERATORS}

Arazy, Fisher, and Peetre prove the following theorem in [2, Theorem 16]. 
TheOREM A. Let $\mu$ be a positive measure on $U, 0<p<\infty$. Then,

$$
\int_{U} \frac{d \mu(z)}{\left(1-|z|^{2}\right)^{p}}<\infty
$$

if and only if there is a positive constant $c$ such that

$$
\int_{U}\left|f^{\prime}(z)\right|^{p} d \mu(z) \leqslant c\|f\|_{B}
$$

for all $f \in \mathcal{B}$.

Note. The proof of Theorem A can be used to show that a similar result holds for a collection of positive measures $\left\{\mu_{q}: q \in U\right\}$. That is, if $0<p<\infty$, then

$$
\sup _{q \in U} \int_{U} \frac{d \mu_{q}}{\left(1-|z|^{2}\right)^{p}}<\infty
$$

if and only if

$$
\sup _{q \in U} \int_{U}\left|f^{\prime}(z)\right|^{p} d \mu_{q} \leqslant c\|f\|_{B}
$$

for all $f \in \mathcal{B}$.

These results, along with a non-univalent change of variables, yield the following characterisations of bounded composition operators from the Bloch space to BMOA.

Proposition 3.1. Let $\phi$ be a holomorphic self-map of $U . C_{\phi}: \mathcal{B} \rightarrow$ BMOA is a bounded operator if and only if

$$
\sup _{q \in U} \int_{U} \frac{N(w, q, \phi)}{\left(1-|w|^{2}\right)^{2}} d A(w)=\sup _{q \in U} \int_{U} \frac{\left|\phi^{\prime}(z)\right|^{2}\left(1-\left|\alpha_{q}(z)\right|^{2}\right)}{\left(1-|\phi(z)|^{2}\right)^{2}} d A(z)<\infty .
$$

We would like to use the following operator theoretic wisdom:

If a "big-oh" condition characterises the boundness of an operator then the corresponding "little-oh" condition should characterise the compactness of the operator.

Proposition 3.2. Let $\phi$ be a holomorphic self-map of $U$. If

$$
\lim _{|q| \rightarrow 1} \int_{U} \frac{\left|\phi^{\prime}(z)\right|^{2}\left(1-\left|\alpha_{q}(z)\right|^{2}\right)}{\left(1-|\phi(z)|^{2}\right)^{2}} d A(z)=0
$$

then $C_{\phi}: \mathcal{B} \rightarrow$ BMOA is a compact operator.

Proof: Let $b(\mathcal{B})$ be the unit ball in $\mathcal{B}$ and $\left(g_{n}\right) \subset b(\mathcal{B})$. Since $\left(g_{n}\right)$ is a normal family in $D$ we can extract a subsequence $\left(g_{n_{k}}\right)$ converging uniformly on compact subsets of $U$ to some function $g \in b(\mathcal{B})$. Then the sequence $\left(f_{k}\right), f_{k}(z)=g_{n_{k}}(z)-g(z)$, converges uniformly to 0 on compact subsets of $U$. Thus for the compactness of the operator 
$C_{\phi}: \mathcal{B} \rightarrow$ BMOA it is enough to prove that $\lim _{k \rightarrow \infty}\left\|f_{k} \circ \phi\right\|_{*}=0$ on every sequence $\left(f_{k}\right) \in \mathcal{B}$ converging to 0 uniformly on compact subsets of $U$.

Let $\left(f_{n}\right)$ be a bounded sequence in $\mathcal{B}$ such that $f_{n} \rightarrow 0$ uniformly on compact sets as $n \rightarrow \infty$. Let $\varepsilon>0$ be given. Then by our hypothesis there is a $\delta>0$ such that if $|q|>1-\delta$ then

$$
\int_{U} \frac{\left|\phi^{\prime}(z)\right|^{2}\left(1-\left|\alpha_{q}(z)\right|^{2}\right)}{\left(1-|\phi(z)|^{2}\right)^{2}}<\varepsilon .
$$

Fix $q \in U$ such that $|q|>1-\delta$. Then

$$
\begin{aligned}
& \int_{U}\left|f_{n}^{\prime}(\phi(z))\right|^{2}\left|\phi^{\prime}(z)\right|^{2}\left(1-\left|\alpha_{q}(z)\right|^{2}\right) d A(z) \\
& \quad=\int_{U}\left|f_{n}^{\prime}(\phi(z))\right|^{2}\left(1-|\phi(z)|^{2}\right)^{2} \frac{\left|\phi^{\prime}(z)\right|^{2}\left(1-\left|\alpha_{q}(z)\right|^{2}\right)}{\left(1-|\phi(z)|^{2}\right)^{2}} d A(z) \\
& \quad \leqslant\left\|f_{n}\right\|_{\mathcal{B}}^{2} \int_{U} \frac{\left|\phi^{\prime}(z)\right|^{2}\left(1-\left|\alpha_{q}(z)\right|^{2}\right)}{\left(1-|\phi(z)|^{2}\right)^{2}} d A(z) \quad(\text { by (11)) } \\
& \quad \leqslant \text { const. } \varepsilon .
\end{aligned}
$$

If $|q| \leqslant 1-\delta$ then

$$
\begin{aligned}
& \int_{U}\left|f_{n}^{\prime}(\phi(z))\right|^{2}\left|\phi^{\prime}(z)\right|^{2}\left(1-\left|\alpha_{q}(z)\right|^{2}\right) d A(z) \\
& \quad=\int_{U}\left|f_{n}^{\prime}(\phi(z))\right|^{2}\left|\phi^{\prime}(z)\right|^{2} \frac{\left(1-|q|^{2}\right)\left(1-|z|^{2}\right)}{|1-\bar{q} z|^{2}} d A(z) \quad(\text { by }(2)) \\
& \quad \leqslant \text { const. } \int_{U}\left|f_{n}^{\prime}(\phi(z))\right|^{2}\left|\phi^{\prime}(z)\right|^{2}\left(1-|z|^{2}\right) d A(z) \quad\left(\frac{1-|q|^{2}}{|1-\bar{q} z|^{2}} \leqslant \frac{2}{\delta}\right) .
\end{aligned}
$$

Since

$$
\begin{gathered}
\int_{U} \frac{\left|\phi^{\prime}(z)\right|^{2}\left(1-|z|^{2}\right)}{\left(1-|\phi(z)|^{2}\right)^{2}}<\infty, \\
\lim _{h \rightarrow 0} \int_{|\phi(z)|>1-h} \frac{\left|\phi^{\prime}(z)\right|^{2}\left(1-|z|^{2}\right)}{\left(1-|\phi(z)|^{2}\right)^{2}} d A(z)=0 .
\end{gathered}
$$

Therefore without loss of generality

$$
\int_{|\phi(z)|>1-\delta} \frac{\left|\phi^{\prime}(z)\right|^{2}\left(1-|z|^{2}\right)}{\left(1-|\phi(z)|^{2}\right)^{2}}<\varepsilon .
$$


Using (14) it is easy to see that

$$
\begin{aligned}
\int_{U} \mid f_{n}^{\prime} & \left.(\phi(z))\right|^{2}\left|\phi^{\prime}(z)\right|^{2}\left(1-|z|^{2}\right) d A(z) \\
& =\int_{|\phi(z)|>1-\delta}+\int_{|\phi(z)| \leqslant 1-\delta}\left|f_{n}^{\prime}(\phi(z))\right|^{2}\left|\phi^{\prime}(z)\right|^{2}\left(1-|z|^{2}\right) d A(z) \\
& <\text { const. } \varepsilon
\end{aligned}
$$

for $n$ large. Then (12), (13), and (15) yield

$$
\sup _{q \in U} \int_{U}\left|f_{n}^{\prime}(\phi(z))\right|^{2}\left|\phi^{\prime}(z)\right|^{2}\left(1-\left|\alpha_{q}(z)\right|^{2}\right) d A(z)<\text { const. } \varepsilon \text {. }
$$

Therefore by (9), $\left\|C_{\phi} f_{n}\right\|_{*}<$ const. $\varepsilon$. Thus, $\left\|C_{\phi} f_{n}\right\|_{*} \rightarrow 0$ as $n \rightarrow \infty$, and it implies that $C_{\phi}: \mathcal{B} \rightarrow \mathrm{BMOA}$ is a compact operator. This finishes the proof of the proposition.

\section{BLOCH-TO-VMOA COMPOSITION OPERATORS}

LEMMA 4.1. Let $X$ be a Möbius invariant Banach subspace of $\mathcal{B}$. Then,

1. Every bounded sequence $\left(f_{n}\right)$ in $X$ is uniformly bounded on compact sets.

2. For any sequence $\left(f_{n}\right)$ on $X$ such that $\left\|f_{n}\right\|_{X} \rightarrow 0, f_{n}-f_{n}(0) \rightarrow 0$ uniformly on compact sets.

ProOF: In [22, p.82] it was shown that a Bloch function can grow at most as fast as $\log 1 /(1-|z|)$, that is

$$
\begin{aligned}
\left|f_{n}(z)-f_{n}(0)\right| & \leqslant \text { const. }\left\|f_{n}\right\|_{\mathcal{B}} \log \frac{1}{1-|z|} \\
& \leqslant \text { const. }\left\|f_{n}\right\|_{X} \log \frac{1}{1-|z|}
\end{aligned}
$$

Hence the result follows.

Next we give a characterisation of compact composition operators whose range is a subset of VMOA.

THEOREM 4.2. Let $\phi$ be a holomorphic self-map of $U$, and $X$ a Möbius invariant Banach space. Then $C_{\phi}: X \rightarrow$ VMOA is a compact operator if and only if

$$
\lim _{|q| \rightarrow 1} \sup _{\substack{\|f\|_{X}<1 \\ f \in X}} \int_{U}\left|f^{\prime}(\phi(z))\right|^{2}\left|\phi^{\prime}(z)\right|^{2}\left(1-\left|\alpha_{q}(z)\right|^{2}\right) d A(z)=0 .
$$

Proof: First suppose that $C_{\phi}: X \rightarrow$ VMOA is a compact operator. Then $A=$ cl $\left(\left\{f \circ \phi \in\right.\right.$ VMOA : $\left.\left.\|f\|_{X}<1\right\}\right)$, the VMOA closure of the image under $C_{\phi}$ of the unit ball of $X$, is a compact subset of VMOA. Let $\varepsilon>0$ be given. Then there is a finite subset of $X, B=\left\{f_{1}, f_{2}, f_{3}, \ldots, f_{N}\right\}$, such that each function in $A$ lies at most $\varepsilon$ distant from $B$. That is, if $g \in A$ then there exists $j \in J=\{1,2,3, \ldots, N\}$ such that

$$
\left\|g-f_{j} \circ \phi\right\|_{*}<\frac{\varepsilon}{4}
$$


Since $\left\{f_{j} \circ \phi: j \in J\right\} \subset \mathrm{VMOA}$, there exists a $\delta>0$ such that for all $j \in J$ and $|q|>1-\delta$,

$$
\int_{U}\left|\left(f_{j} \circ \phi\right)^{\prime}(z)\right|^{2}\left(1-\left|\alpha_{q}(z)\right|^{2}\right) d A(z)<\frac{\varepsilon}{4}
$$

By (16) and (17) we obtain that for each $|q|>1-\delta$ and $f \in X$ such that $\|f\|_{X}<1$ there exists $j \in J$ such that

$$
\begin{aligned}
\int_{U}\left|(f \circ \phi)^{\prime}(z)\right|^{2}\left(1-\left|\alpha_{q}(z)\right|^{2}\right) d A(z) \\
\leqslant 2 \int_{U}\left|\left(f \circ \phi-f_{j} \circ \phi\right)^{\prime}\right|^{2}\left(1-\left|\alpha_{q}(z)\right|^{2}\right) d A(z) \\
\quad+2 \int_{U}\left|\left(f_{j} \circ \phi\right)^{\prime}(z)\right|^{2}\left(1-\left|\alpha_{q}(z)\right|^{2}\right) d A(z) \\
<2 \frac{\varepsilon}{4}+2 \frac{\varepsilon}{4}=\varepsilon .
\end{aligned}
$$

This proves one direction.

In order to prove the converse, let $\left(f_{n}\right)$ be a sequence in the unit ball of $X$. By Lemma 4.1 and Montel's Theorem there exists a subsequence $n_{1}<n_{2}<\ldots$ and a function $g$ holomorphic on $U$ such that $f_{n_{k}} \rightarrow g$ uniformly on compact sets, as $k \rightarrow \infty$. By our hypothesis and Fatou's Lemma it is easy to see that $C_{\phi} g \in$ VMOA. We shall show that $\left\|C_{\phi}\left(f_{n_{k}}-g\right)\right\|_{*} \rightarrow 0$, as $k \rightarrow \infty$. In order to simplify the notation we shall assume, without loss of generality, that we are given a sequence $\left(f_{n}\right)$ in the unit ball of $X$ such that $f_{n} \rightarrow 0$ uniformly on compact sets, as $n \rightarrow \infty$. We shall show that

$$
\lim _{n \rightarrow \infty}\left\|C_{\phi} f_{n}\right\|_{*}=0 \text {. }
$$

To prove (18) we shall use the equivalent BMOA norm as given by (6). Thus, our hypothesis is equivalent to

$$
\lim _{h \rightarrow 0} \sup _{\substack{\theta \in\left\{(0,2 \pi) \\\left\{\delta \in X:\|f \mid\|_{X}<1\right\}\right.}} \frac{1}{h} \int_{S(h, \theta)}\left|(f \circ \phi)^{\prime}(z)\right|^{2}\left(1-|z|^{2}\right) d A(z)=0 .
$$

Let $\varepsilon>0$ be given. By (19), there exists a $\delta>0$ such that if $n \in \mathbb{N}, \theta \in[0,2 \pi)$, and $h<\delta$ then

$$
\frac{1}{h} \int_{S(h, \theta)}\left|\left(f_{n} \circ \phi\right)^{\prime}(z)\right|^{2}\left(1-|z|^{2}\right) d A(z)<\varepsilon .
$$

Fix $h_{0}<\delta, \theta \in[0,2 \pi), n \in \mathbb{N}$, and $h \geqslant \delta$. It is easy to see that there exists $\left\{\theta_{1}, \theta_{2}, \ldots, \theta_{N}\right\} \subset[0,2 \pi)$ such that $S(h, \theta)$ is the union of the sets $\left\{S\left(h_{0}, \theta_{j}\right): j=\right.$ $1,2, \ldots, N\}$ and $K$, a compact subset of $U$. Hence,

$$
\begin{aligned}
& \frac{1}{h} \int_{S(h, \theta)}\left|\left(f_{n} \circ \phi\right)^{\prime}(z)\right|^{2}\left(1-|z|^{2}\right) d A(z) \\
& \quad \leqslant \sum_{j=1}^{N} \frac{1}{h_{0}} \int_{S\left(h_{0}, \theta_{j}\right)}+\frac{1}{h_{0}} \int_{K}\left|\left(f_{n} \circ \phi\right)^{\prime}(z)\right|^{2}\left(1-|z|^{2}\right) d A(z) \\
& \quad=I+I I .
\end{aligned}
$$


Since $f_{n}^{\prime} \rightarrow 0$ uniformly on $K$, as $n \rightarrow \infty$, there exists an $N \in \mathbb{N}$ such that for $n \geqslant N$

$$
I I \leqslant \frac{\varepsilon}{h_{0}} \int_{K}\left(1-|z|^{2}\right) d A(z) \leqslant \text { const. } \varepsilon .
$$

Moreover (20) yields,

$$
I \leqslant \sum_{j=1}^{N} \varepsilon=\text { const. } \varepsilon .
$$

Hence (20), (21), (22), and (23) yield (18). Thus a similar argument to that described in Proposition 3.2 yields that $C_{\phi}: X \rightarrow$ VMOA is a compact operator.

NoTE. Bourdon, Cima and Matheson have obtained a characterisation of compactness of $C_{\phi}:$ VMOA $\rightarrow$ VMOA that is similar to the theorem above (see [5, Theorem 3.5]).

Next we show that the sufficient condition of compactness of $C_{\phi}: \mathcal{B} \rightarrow$ BMOA in Proposition 3.2 is also necessary for the compactness of $C_{\phi}: \mathcal{B} \rightarrow$ VMOA. We shall use Khintchine's inequality for gap series (as done by Arazy, Fisher, and Peetre in [2, Theorem 16]), and Theorem 4.2.

THEOREM 4.3. Let $\phi$ be a holomorphic self-map of $U$. Then the following are equivalent:

1. $C_{\phi}: \mathcal{B} \rightarrow$ VMOA is a compact operator.

2.

$$
\lim _{|q| \rightarrow 1} \int_{U} \frac{\left|\phi^{\prime}(z)\right|^{2}\left(1-\left|\alpha_{q}(z)\right|^{2}\right)}{\left(1-|\phi(z)|^{2}\right)^{2}} d A(z)=0
$$

Proof: First, suppose that (1) holds. Then by Theorem 4.2 and since

$$
f_{\theta}(z)=\sum_{n=0}^{\infty}\left(e^{i \theta} z\right)^{2^{n}} \in \mathcal{B}
$$

for all $\theta \in[0,2 \pi)$ (see $[1$, Lemma 2.1]),

$$
\lim _{|q| \rightarrow 1} \sup _{\theta \in[0,2 \pi)} \int_{U}\left|\sum_{n=0}^{\infty} 2^{n}\left(e^{i \theta} w\right)^{2^{n}-1}\right|^{2} N(w, q, \phi) d A(w)=0 .
$$

Let $\varepsilon>0$ be given. Then there exists a $\delta>0$ such that for any $q \in U$ with $|q|>1-\delta$ and any $\theta \in[0,2 \pi)$,

$$
A_{\theta} \stackrel{\text { def. }}{=} \int_{U}\left|\sum_{n=0}^{\infty} 2^{n}\left(e^{i \theta} w\right)^{2^{n}-1}\right|^{2} N(w, q, \phi) d A(w)<\varepsilon .
$$

Upon integrating (24) with respect to $(d \theta / 2 \pi)$ and using Fubini's Theorem, we obtain

$$
\int_{0}^{2 \pi} A_{\theta} \frac{d \theta}{2 \pi}=\int_{U}\left\{\int_{0}^{2 \pi}\left|\sum_{n=0}^{\infty} 2^{n} e^{i \theta\left(2^{n}-1\right)} w^{2^{n}-1}\right|^{2} \frac{d \theta}{2 \pi}\right\} N(w, q, \phi) d A(w) \leqslant \varepsilon .
$$


Khintchine's inequality (see [23, Theorem V.8.4]) for gap series yields that for any positive integer $N$

$$
\int_{0}^{2 \pi}\left|\sum_{n=0}^{N} 2^{n} e^{i \theta\left(2^{n}-1\right)} w^{2^{n}-1}\right|^{2} \frac{d \theta}{2 \pi} \sim \sum_{n=0}^{N} 2^{2 n}|w|^{2^{n+1}-2} .
$$

Therefore (25) and (26) imply that

$$
\int_{0}^{2 \pi} A_{\theta} \frac{d \theta}{2 \pi} \sim \int_{U}\left\{\sum_{n=0}^{\infty} 2^{2 n}|w|^{2^{n+1}-2}\right\} N(w, q, \phi) d A(w) .
$$

It is shown in $[\mathbf{2}$, Theorem 16] that

$$
\sum_{n=0}^{\infty} 2^{2 n}|w|^{2^{n+1}} \geqslant \frac{\text { const. }}{\left(1-|w|^{2}\right)^{2}},
$$

for any $w \in U$ such that $|w| \geqslant 1 / 2$. Hence (25), (27), and (28) yield

$$
\int_{U} \frac{N(w, q, \phi)}{\left(1-|w|^{2}\right)^{2}} d A(w) \leqslant \text { const. } \int_{0}^{2 \pi} A_{\theta} \frac{d \theta}{2 \pi}<\text { const. } \varepsilon,
$$

for any $q \in U$ with $|q|>1-\delta$, and any $\varepsilon>0$. Thus (29) yields (2).

Conversely, suppose that (2) holds. Fix $f$ in the unit ball of the Bloch space. Then,

$$
\begin{aligned}
\int_{U}\left|f^{\prime}(\phi(z))\right|^{2}\left|\phi^{\prime}(z)\right|^{2}\left(1-\left|\alpha_{q}(z)\right|^{2}\right) d A(z) & \leqslant\|f\|_{\mathcal{B}}^{2} \int_{U} \frac{\left|\phi^{\prime}(z)\right|^{2}\left(1-\left|\alpha_{q}(z)\right|^{2}\right)}{\left(1-|\phi(z)|^{2}\right)^{2}} d A(z) \\
& \leqslant \int_{U} \frac{\left|\phi^{\prime}(z)\right|^{2}\left(1-\left|\alpha_{q}(z)\right|^{2}\right)}{\left(1-|\phi(z)|^{2}\right)^{2}} d A(z) .
\end{aligned}
$$

The righthand side of the above inequality tends to 0 , as $|q| \rightarrow 1$, by our hypothesis. Hence Theorem 4.2 yields that $C_{\phi}: \mathcal{B} \rightarrow$ VMOA is a compact operator. This finishes the proof of the theorem.

5. Similarity OF COMPACTNESS CONDITIONS FOR ThE BLOCH SPACE, BMOA AND VMOA

LEMma 5.1. If $\phi$ is a holomorphic self-map of $U$ such that $\|\phi\|_{\infty}<1$ then $C_{\phi}$ is compact on BMOA.

PROOF Let $\left(f_{n}\right)$ be a bounded sequence in BMOA such that $f_{n} \rightarrow 0$ uniformly on compact subsets of $U$. Suppose that $\varepsilon>0$ is given. Since $\overline{\phi(U)}$ is a compact subset of $U$, there exists a positive integer $N$ such that if $n \geqslant N$ then $\left|f_{n}^{\prime}(\phi(z))\right|^{2}<\varepsilon$, for all $z \in U$. Then by (8),

$$
\left\|C_{\phi} f_{n}\right\|_{*}^{2}<\varepsilon\|\phi\|_{*}^{2}<\text { const. } \varepsilon
$$


Thus, $\left\|C_{\phi} f_{n}\right\|_{*} \rightarrow 0$, as $n \rightarrow \infty$, and hence $C_{\phi}$ is a compact operator on BMOA.

There are symbols $\phi$ such that $C_{\phi}$ is compact on BMOA but not on VMOA. For example, consider the self-map $\phi(z)=\exp \{(z+1) /(z-1)\} / 2$. Since $\|\phi\|_{\infty}<1$, Lemma 5.1 yields that $C_{\phi}$ is a compact operator on BMOA. Moreover since $\phi \notin \mathcal{B}_{0}$, the operator $C_{\phi}$ is not even bounded on VMOA (see [2, Theorem 12]).

If $\phi \in \mathrm{VMOA}$ then compactness of $C_{\phi}$ on BMOA implies the compactness of $C_{\phi}$ on VMOA. If $T$ is a compact operator on a Banach space $X$, and $Y$ is an invariant subspace of $X$ such that $T: Y \rightarrow Y$ is bounded, then $T: Y \rightarrow Y$ is a compact operator as well. Thus we obtain the following proposition.

PROPOSITION 5.2. Let $\phi$ be a holomorphic self-map of $U$. Then,

1. If $\phi \in \mathrm{VMOA}$ and $C_{\phi}: \mathrm{BMOA} \rightarrow \mathrm{BMOA}$ is a compact operator then $C_{\phi}:$ VMOA $\rightarrow$ VMOA is a compact operator.

2. If $\phi \in \mathcal{B}_{0}$ and $C_{\phi}: \mathcal{B} \rightarrow \mathcal{B}$ is a compact operator then $C_{\phi}: \mathcal{B}_{0} \rightarrow \mathcal{B}_{0}$ is a compact operator.

Next we show that composition operators $C_{\phi}: \mathcal{B} \rightarrow \mathrm{BMOA}$ and $C_{\phi}: \mathcal{B} \rightarrow$ VMOA, where the symbol is a boundedly valent holomorphic function whose image lies inside a polygon inscribed in the unit circle, are compact if and only if they are compact on the Bloch space. We shall use Propositions 3.2, 5.2, and the following theorem of Pommerenke [13, Satz 1].

THEOREM B. Let $f$ be a holomorphic function on $U$ such that

$$
\sup _{w_{0} \in \mathbf{C}} \int_{\left|w-w_{0}\right|<1} \eta(f, w) d A(w)<\infty
$$

Then,

$$
f \in \mathrm{BMOA} \Leftrightarrow f \in \mathcal{B}, f \in \mathrm{VMOA} \Leftrightarrow f \in \mathcal{B}_{0} .
$$

A non-tangential approach region $\Omega_{\alpha}(0<\alpha<1)$ in $U$, with vertex $\zeta \in \partial U$, is the convex hull of $D(0, \alpha) \cup\{\zeta\}$ minus the point $\zeta$. The exact shape of the region is not relevant. The important fact that we shall use in the theorem below is that there exists $0<r<1$ and $c>0$ such that if $z \in \Omega_{\alpha}$ and $|\zeta-z|<r$, then

$$
|\zeta-z| \leqslant c\left(1-|z|^{2}\right) \text {. }
$$

THEOREM 5.3. Let $\phi$ be a boundedly valent holomorphic self-map of $U$ such that $\phi(U)$ lies inside a polygon inscribed in the unit circle. Then the following are equivalent:

1. $C_{\phi}: \mathcal{B} \rightarrow$ VMOA is a compact operator.

2. $C_{\phi}: \mathcal{B} \rightarrow \mathrm{BMOA}$ is a compact operator.

3. $C_{\phi}: \mathrm{BMOA} \rightarrow \mathrm{BMOA}$ is a compact operator.

4. $C_{\phi}: \mathcal{B} \rightarrow \mathcal{B}$ is a compact operator. 
5. $C_{\phi}: \mathcal{B}_{0} \rightarrow \mathcal{B}_{0}$ is a compact operator.

6. $C_{\phi}: \mathrm{VMOA} \rightarrow \mathrm{VMOA}$ is a compact operator.

Proof: $(1) \Rightarrow(2) \Rightarrow(3)$ and $(1) \Rightarrow(4)$ are clear.

Since any boundedly valent holomorphic self-map of $U$ belongs to VMOA, Proposition 5.2 yields $(4) \Rightarrow(5)$ and $(3) \Rightarrow(6)$.

$(5) \Rightarrow(1)$. By Madigan and Matheson's Theorem 1 (see [10]) $C_{\phi}$ is a compact operator on the little Bloch space if and only if

$$
\lim _{|z| \rightarrow 1} \frac{\left|\phi^{\prime}(z)\right|\left(1-|z|^{2}\right)}{1-|\phi(z)|^{2}}=0 .
$$

It follows that $\log 1 /(w-\phi) \in \mathcal{B}_{0}$ for each $w \in \partial U$. By Theorem B each boundedly valent function in $\mathcal{B}_{0}$ must belong to VMOA, hence $\log 1 /(w-\phi) \in \mathrm{VMOA}$ for each $w \in \partial U$. Thus

$$
\lim _{|q| \rightarrow 1} \int_{U}\left|(\log 1 /(w-\phi(z)))^{\prime}\right|^{2}\left(1-\left|\alpha_{q}(z)\right|^{2}\right) d A(z)=0
$$

hence

$$
\lim _{|q| \rightarrow 1} \int_{U} \frac{\left|\phi^{\prime}(z)\right|^{2}\left(1-\left|\alpha_{q}(z)\right|^{2}\right)}{|w-\phi(z)|^{2}} d A(z)=0,
$$

for each $w \in \partial U$.

Let $\left\{w_{j}: 1 \leqslant j \leqslant n\right\}$ be the vertices of the inscribed polygon containing $\phi(U)$. Break the unit disc up into a compact set $K$ and finitely many regions

$$
E_{j}=\left\{z \in U:\left|w_{j}-\phi(z)\right|<r\right\}
$$

where $r$ is chosen so that the regions are disjoint, and so that

$$
\left|w_{j}-\phi(z)\right| \leqslant \text { const. }\left(1-|\phi(z)|^{2}\right)
$$

for each $z \in E_{j}$ and each $j$. Then for each $q \in U$,

$$
\int_{E_{j}} \frac{\left|\phi^{\prime}(z)\right|^{2}\left(1-\left|\alpha_{q}(z)\right|^{2}\right)}{\left(1-|\phi(z)|^{2}\right)^{2}} d A(z) \leqslant \text { const. } \int_{E_{j}} \frac{\left|\phi^{\prime}(z)\right|^{2}\left(1-\left|\alpha_{q}(z)\right|^{2}\right)}{\left|w_{j}-\phi(z)\right|^{2}} d A(z)
$$

Hence

$$
\begin{aligned}
& \int_{U} \frac{\left|\phi^{\prime}(z)\right|^{2}\left(1-\left|\alpha_{q}(z)\right|^{2}\right)}{\left(1-|\phi(z)|^{2}\right)^{2}} d A(z)=\sum_{j=1}^{n} \int_{E_{j}}+\int_{K} \frac{\left|\phi^{\prime}(z)\right|^{2}\left(1-\left|\alpha_{q}(z)\right|^{2}\right)}{\left(1-|\phi(z)|^{2}\right)^{2}} d A(z) \\
& \leqslant \text { const. } \sum_{j=1}^{n} \int_{E_{j}} \frac{\left|\phi^{\prime}(z)\right|^{2}\left(1-\left|\alpha_{q}(z)\right|^{2}\right)}{\left|w_{j}-\phi(z)\right|^{2}} d A(z) \\
& + \text { const. } \int_{U}\left|\phi^{\prime}(z)\right|^{2}\left(1-\left|\alpha_{q}(z)\right|^{2}\right) d A(z) \text {, }
\end{aligned}
$$


for all $q \in U$.

Any boundedly valent holomorphic self-map of $U$ belongs to VMOA. Hence (31) and (32) imply that

$$
\lim _{-|q| \rightarrow 1} \int_{U} \frac{\left|\phi^{\prime}(z)\right|^{2}\left(1-\left|\alpha_{q}(z)\right|^{2}\right)}{\left(1-|\phi(z)|^{2}\right)^{2}} d A(z)=0 .
$$

By (33) and Theorem 4.3 we obtain that $C_{\phi}: \mathcal{B} \rightarrow$ VMOA is a compact operator.

If (6) holds, that is $C_{\phi}: \mathrm{VMOA} \rightarrow \mathrm{VMOA}$ is a compact operator, then $C_{\phi}$ is weakly compact on VMOA. Hence by Theorem VI 5.5 in [7, p.189], $C_{\phi}(\mathrm{BMOA}) \subset$ VMOA. Thus $\log 1 /(w-\phi(z)) \in$ VMOA $(w \in \partial U)$. Thus by the proof of $(5) \Rightarrow(1)$ we obtain $(6) \Rightarrow(1)$. This finishes the proof of the theorem.

Note. W. Smith recently proved (see [19, Theorem 4.1]) that statements (3) through (6) of the theorem above are equivalent for any univalent self-map of $U$. The proof of $(5) \Rightarrow(1)$ of the theorem above has the corollary below.

Madigan and Matheson showed (see [10, Theorem 3, p.2683])) that every weakly compact composition operator $C_{\phi}$ on $\mathcal{B}_{0}$ is compact. The second author in her thesis (see [20]), and Bourdon, Cima and Matheson (see [5]) asked a similar question for composition operators in VMOA. We show below that if $C_{\phi}$ is weakly compact on VMOA then it is compact, if $\phi(U)$ lies inside a polygon inscribed in the unit circle.

COROLlary 5.4. Let $\phi$ be a bounded holomorphic self-map of $U$ such that $\phi(U)$ lies inside a polygon inscribed in the unit circle. Then the following are equivalent:

1. $C_{\phi}: \mathrm{VMOA} \rightarrow \mathrm{VMOA}$ is a weakly compact operator.

2. $C_{\phi}: \mathrm{VMOA} \rightarrow \mathrm{VMOA}$ is a compact operator.

Proof: $(1) \Rightarrow(2)$. The operator $C_{\phi}: \mathrm{VMOA} \rightarrow \mathrm{VMOA}$ is weakly compact if and only if the closure of the image under $C_{\phi}$ of the unit ball in VMOA is a weakly compact subset of VMOA. By Gantmacher's theorem (see $[8, \mathrm{p} .482]$ ) the operator $C_{\phi}:$ VMOA $\rightarrow$ VMOA is weakly compact if and if $C_{\phi}^{* *}\left(\mathrm{VMOA}^{* *}\right) \subseteq \mathrm{VMOA}$. Since $\mathrm{VMOA}^{* *}$ is BMOA, $C_{\phi}: \mathrm{VMOA} \rightarrow \mathrm{VMOA}$ is weakly compact if and only if $C_{\phi}(\mathrm{BMOA}) \subseteq \mathrm{VMOA}$. The functions $\log 1 /(w-z)$ belong to BMOA, for each $w \in \partial U$. Thus, $\log 1 /(w-\phi) \epsilon$ VMOA, for each $w \in \partial U$. It now follows from the proof of $(5) \Rightarrow(1)$ of Theorem 5.3 that $C_{\phi}: \mathcal{B} \rightarrow \mathrm{VMOA}$ is a compact operator (note that we do not need to assume that $\phi$ is boundedly valent. It was needed in the proof of $(5) \Rightarrow(1)$ of Theorem 5.3 so that we may conclude that $\phi \in \mathrm{VMOA}$. Here we already know that). This implies that $C_{\phi}: \mathrm{VMOA} \rightarrow \mathrm{VMOA}$ is a compact operator.

$(2) \Rightarrow(1)$ is clear. 
DEFINITION 5.5. A region $G \subset U$ is said to have a nontangential cusp at $\zeta \in \partial U$ if

$$
\lim _{\substack{z \rightarrow 1 \\ z \in G}} \frac{|\operatorname{Im} z|}{|1-z|}=0 .
$$

Theorem 5.3 and Madigan and Matheson's Theorem 5 (see [10, p.2685]) yield the corollary below:

CoROLlary 5.6. If $\phi$ is a univalent self-map of $U$ such that $\phi(U)$ has finitely many points of contact with $\partial U$ and such that at each of these points $\phi(U)$ has a nontangential cusp, then $C_{\phi}$ is a compact operator on BMOA and on VMOA.

\section{HyPERBolic VMOA FUNCTIONS}

Choe, Ramey and Ullrich [6] proved that a function $\phi \in B$ induces the bounded Bloch-to-BMOA composition operator $C_{\phi}$ if and only if $\phi$ is a hyperbolic BMOA function $\left(\phi \in \mathrm{BMOA}_{h}\right)[21]$, that is,

$$
\sup _{q \in U} \int_{\partial U} \sigma\left(\phi \circ \alpha_{q}(z), \phi(q)\right)|d z|<\infty
$$

By Yamashita [21], the necessary and sufficient condition for a function $\phi \in B$ to be in $\mathrm{BMOA}_{h}$ is

$$
\sup _{q \in U} \int_{U}\left(\frac{\left|\phi^{\prime}(z)\right|}{1-|\phi(z)|^{2}}\right)^{2} \ln \left|\frac{1-\bar{q} z}{q-z}\right| d x d y<\infty .
$$

By the equivalence $\log 1 /|z| \sim 1-|z|^{2}$ for $z$ away from the origin, condition (34) is equivalent to (10) (Proposition 3.2).

We say that $\phi \in B$ is a hyperbolic VMOA function, that is, $\phi \in \mathrm{VMOA}_{h}$, if

$$
\lim _{|q| \rightarrow 1} \int_{\partial U} \sigma\left(\phi \circ \alpha_{q}(z), \phi(q)\right)|d z|=0
$$

By the equivalence $(\log (1+x) /(1-x)) / 2 \sim \log \left(1-x^{2}\right)$ as $x \rightarrow 0$, condition (35) is equivalent to

$$
\lim _{|q| \rightarrow 1} \int_{\partial U} \log \left(1-\left|\frac{\phi \circ \alpha_{q}(z)-\phi(q)}{1-\phi \circ \alpha_{q}(z) \overline{\phi(q)}}\right|^{2}\right)|d z|=0 .
$$

THEOREM 6.1. The following conditions are equivalent

(1) $\phi \in \mathrm{VMOA}_{h}$.

(2) $\lim _{|q| \rightarrow 1} \int_{U}\left(\frac{\left|\phi^{\prime}(z)\right|}{1-|\phi(z)|^{2}}\right)^{2}\left(1-\left|\alpha_{q}(z)\right|^{2}\right) d x d y=0$;

(3) $C_{\phi}: \mathcal{B} \rightarrow$ VMOA is a compact operator. 
Proof: The equivalence of (1) and (2) follows from equivalence of (36) and (2) which we prove.

Let

$$
\Lambda(\phi, z, q)=-\frac{1}{2} \ln \left(1-\left|\frac{\phi(z)-\phi(q)}{1-\overline{\phi(q)} \phi(z)}\right|^{2}\right)
$$

Since

$$
\Delta \Lambda(\phi, z, q)=2\left(\frac{\left|\phi^{\prime}(z)\right|}{1-|\phi(z)|^{2}}\right)^{2}
$$

and by the Green formula [12]

$$
\begin{aligned}
-\frac{1}{2} \int_{\partial U} \ln \left(1-\rho^{2}\left(\phi_{q}(z), \phi(q)\right)|d z|\right) & =\int_{U}\left(\frac{\left|\phi^{\prime}(z)\right|}{1-|\phi(z)|^{2}}\right)^{2} \log \left|\frac{1-\bar{q} z}{z-q}\right| d x d y \\
& \sim \int_{U}\left(\frac{\left|\phi^{\prime}(z)\right|}{1-|\phi(z)|^{2}}\right)^{2}\left(1-\left|\alpha_{q}(z)\right|^{2}\right) d x d y
\end{aligned}
$$

as $|q| \rightarrow 1$. Thus the equivalence of (36) and (2) becomes clear.

$(2) \Leftrightarrow(3)$ follows from theorem 4.3.

The classes $B_{p}^{h}$ of hyperbolic Besov functions, $1<p<\infty$, were defined in [11]. Hyperbolic Besov class $B_{p}^{h}, 1<p<\infty$, contains functions $\phi \in B$ such that

$$
\|\phi\|_{B_{p}^{h}}=\left(\int_{U}\left(1-|z|^{2}\right)^{p-2}\left(\frac{\left|\phi^{\prime}(z)\right|}{1-|\phi(z)|^{2}}\right)^{p} d x d y\right)^{1 / p}<\infty .
$$

THEOREM 6.2. $B_{p}^{h} \subset \mathrm{VMOA}_{h} \subset B_{0}$ for every $1<p<\infty$.

Proof: Let $\phi \in \mathrm{VMOA}_{h}$. By Theorem 6.1 $C_{\phi}: \mathcal{B} \rightarrow \mathrm{VMOA}$ is a compact operator. Thus, $C_{\phi}: \mathcal{B}_{0} \rightarrow \mathcal{B}_{0}$ is a compact operator. It follows from the characterisation of compactness of the composition operator on $\mathcal{B}_{0}$ that Madigan and Matheson gave (see [10, Theorem 1, p.2681]) that $\phi \in B_{0}$.

Next, let $\phi \in B_{p}^{h}$. For arbitrary $R, 0<R<1$,

$$
\begin{aligned}
\int_{U}\left(\frac{\left|\phi^{\prime}(z)\right|}{1-|\phi(z)|^{2}}\right)^{2}\left(1-\left|\alpha_{q}(z)\right|^{2}\right) d x d y= & \int_{D(q, R)}\left(\frac{\left|\phi^{\prime}(z)\right|}{1-|\phi(z)|^{2}}\right)^{2}\left(1-\left|\alpha_{q}(z)\right|^{2}\right) d x d y \\
& +\int_{U \backslash D(q, R)}\left(\frac{\left|\phi^{\prime}(z)\right|}{1-|\phi(z)|^{2}}\right)^{2}\left(1-\left|\alpha_{q}(z)\right|^{2}\right) d x d y \\
= & I_{1}(q, R)+I_{2}(q, R) .
\end{aligned}
$$

We shall prove that $I_{2}(q, R) \rightarrow 0$ as $R \rightarrow 1$ and $I_{1}(q, R) \rightarrow 0$ as $R \rightarrow 1$ and $|q| \rightarrow 1$. 
$[17]$

Composition operators on Banach spaces

17

Here we take $0<s<1$ and apply the Schwarz-Pick Lemma

$$
\begin{aligned}
I_{2}(q, R) & =\int_{U \backslash D(q, R)}\left(\frac{\left|\phi^{\prime}(z)\right|}{1-|\phi(z)|^{2}}\right)^{2}\left(1-\left|\alpha_{q}(z)\right|^{2}\right) d x d y \\
& =\int_{U \backslash D(0, R)}\left(\frac{\left|\phi_{q}^{\prime}(z)\right|}{1-\left|\phi_{q}(z)\right|^{2}}\right)^{2}\left(1-|z|^{2}\right) d x d y \\
& \leqslant \int_{U \backslash D_{R}}\left(\frac{\left|\phi_{q}^{\prime}(z)\right|}{1-\left|\phi_{q}(z)\right|^{2}}\right)^{s}\left(1-|z|^{2}\right) \frac{1}{\left(1-|z|^{2}\right)^{2-s} d x d y} \\
& =\int_{U \backslash D_{R}}\left(\frac{\left|\phi_{q}^{\prime}(z)\right|}{1-\left|\phi_{q}(z)\right|^{2}}\right)^{s}\left(1-|z|^{2}\right)^{s-1} d x d y \\
& =\int_{U \backslash D_{R}}\left(\frac{\left|\phi_{q}^{\prime}(z)\right|}{1-\left|\phi_{q}(z)\right|^{2}}\right)^{s}\left(1-|z|^{2}\right)^{s(p-2) / p}\left(1-|z|^{2}\right)^{s-1-s(p-2) / p} d x d y \\
& \leqslant\left(\int_{U \backslash D_{R}}\left[\left(\frac{\left|\phi_{q}^{\prime}(z)\right|^{s}}{1-\left|\phi_{q}(z)\right|^{2}}\right)^{s}\left(1-|z|^{2}\right)^{s(p-2) / p}\right]^{p / s} d x d y\right)^{s / p} \\
& \leqslant\|\phi\|_{B_{p}^{h}}^{s} \cdot\left(\int_{U \backslash D_{R}}\left[\left(1-|z|^{2}\right)^{s-1-s(p-2) / p}\right]^{p /(p-s)} d x d y\right)^{(p-s) / p} \\
& \left.\left(1-|z|^{2}\right)^{-1+s /(p-s)} d x d y\right)^{(p-s) / p} \rightarrow 0, \quad R \rightarrow 1 .
\end{aligned}
$$

Now we prove that $I_{1}(q, R) \rightarrow 0$ as $R \rightarrow 1$ and $|q| \rightarrow 1$.

Since $B_{p}^{h} \subset B_{0}$ (see [11]) for any $\varepsilon>0$ there is $R_{0}, 0<R_{0}<1$, such that for any $q$, $R_{0} \leqslant|q|<1$, and for any $R, 0<R<1$,

$$
\sup _{z \in D(q, R)}\left(1-|z|^{2}\right) \frac{\left|\phi^{\prime}(z)\right|}{1-|\phi(z)|^{2}}<\varepsilon .
$$

Then

$$
\begin{aligned}
I_{1}(q, R) & =\int_{D(q, R)}\left(\frac{\left|\phi^{\prime}(z)\right|}{1-|\phi(z)|^{2}}\right)^{2}\left(1-\left|\alpha_{q}(z)\right|^{2}\right) d x d y \\
& \leqslant \int_{D(q, R)}\left(1-|z|^{2}\right)^{2}\left(\frac{\left|\phi^{\prime}(z)\right|}{1-|\phi(z)|^{2}}\right)^{2} \frac{\left(1-\left|\alpha_{q}(z)\right|^{2}\right)}{\left(1-|z|^{2}\right)^{2}} d x d y \\
& \leqslant \varepsilon \int_{D(q, R)} \frac{\left(1-\left|\alpha_{q}(z)\right|^{2}\right)}{\left(1-|z|^{2}\right)^{2}} d x d y
\end{aligned}
$$

https://doi.org/10.1017/S0004972700018426 Published online by Cambridge University Press 


$$
=\varepsilon \int_{D(0, R)} \frac{\left(1-|z|^{2}\right)}{\left(1-|z|^{2}\right)^{2}} d x d y=\varepsilon \cdot \pi \ln \frac{1}{1-R^{2}}
$$

Thus we have

$$
I=I_{1}+I_{2} \leqslant \varepsilon \cdot \text { const. }
$$

and $\phi \in \mathrm{VMOA}_{h}$. The proof is completed.

\section{REFERENCES}

[1] J.M. Anderson, J. Clunie and Ch. Pommerenke, 'On Bloch functions and normal functions', J. Reine Angew. Math. 270 (1974), 12-37.

[2] J. Arazy, S.D. Fisher and J. Peetre, 'Mobius invariant function spaces', J. Reine Angew. Math. 363 (1985), 110-145.

[3] R. Aulaskari, D.A. Stegenga and J. Xiao, 'Some subclasses of BMOA and their characterization in terms of Carleson measures', Rocky Mountain J. Math. 26 (1996), 485-506.

[4] A. Baernstein, 'Analytic functions of bounded mean oscillations', in Aspects of Contemporary Complex Analysis, (D.A. Brannan and J.G. Clunie, Editors) (Academic Press, London, 1980), pp. 3-36.

[5] P.S. Bourdon, J.A. Cima and A. Matheson, 'Compact composition operators on BMOA', Trans. Amer. Math. Soc. (to appear).

[6] B.R. Choe, W. Ramey and D. Ullrich, 'Bloch-to-BMOA pullbacks on the disk', Proc. Amer. Math. Soc. 125 (1997), 2987-2996.

[7] J.B. Conway, A course in functional analysis (Springer-Verlag, Berlin, Heidelberg, New York, 1985).

[8] N. Dunford and J. Schwartz, Linear operators part I: General theory (John Wiley and Sons, New York, 1988).

[9] J.B. Garnett, Bounded analytic functions (Academic Press, New York, 1981).

[10] P.K. Madigan and A. Matheson, 'Compact composition operators on the Bloch space', Trans. Amer. Math. Soc. 347 (1995), 2679-2687.

[11] S. Makhmutov, 'Hyperbolic Besov functions and Bloch-to-Besov composition operators', Hokkaido Math. J. 26 (1997), 699-711.

[12] R. Nevanlinna, Analytic functions, (second edition) (Springer-Verlag, Berlin, Heidelberg, New York, 1970).

[13] C. Pommerenke, 'Schlichte Funktionen und analytische Funktionen von beschränkter mittlerer Oszillation', Comment. Math. Helv. 52 (1977), 591-602.

[14] L.A. Rubel and R.M. Timoney, 'An extremal property of the Bloch space', Proc. Amer. Math. Soc. 75 (1979), 45-49.

[15] D. Sarason, Operator theory on the unit circle (Lecture notes of Virginia Polytechnic Institute and State University, Blacksburg, VA, 1978).

[16] J.H. Shapiro, 'The essential norm of a composition operator', Ann of Math. 125 (1987), 375-404.

[17] J.H. Shapiro, Composition operators and classical function theory (Springer-Verlag, Berlin, Heidelberg, New York, 1993). 
[18] W. Smith and R. Zhao, 'Composition operators mapping into the $Q_{p}$ spaces', Analysis 17 (1997), 239-263.

[19] W. Smith, 'Compactness of composition operators on BMOA', Proc. Amer. Math. Soc. (to appear).

[20] M. Tjani, Compact composition operators on some Möbius invariant Banach spaces, (Thesis) (Michigan State University, 1996).

[21] S. Yamashita, 'Holomorphic functions of hyperbolically bounded mean oscillation', Boll. Un. Mat. Ital. $B(6)$ 5-B (1986), 983-1000.

[22] K. Zhu, Operator theory on function spaces (Marcel Dekker, New York, 1990).

[23] A. Zygmund, Trigonometric series, Volume I, (second edition) (Cambridge University Press, Cambridge, 1959).

Department of Mathematics

Ufa State Aviation Technical University

Ufa 450000

Russia

e-mail: makhm@ugatu.ac.ru
Department of Mathematical Sciences 301 Science Engineering Building

University of Arkansas

Fayetteville AR 72701

United States of America

e-mail: mtjani@comp.uark.edu 